\title{
Propensity Score Methods in Rare Disease: A Demonstration Using Observational Data in Systemic Lupus Erythematosus
}

\author{
Ibrahim Almaghlouth ${ }^{1}$, Eleanor Pullenayegum² ${ }^{2}$ Dafna D. Gladman ${ }^{3}$ D, Murray B. Urowitz ${ }^{3}$ (D), \\ and Sindhu R. Johnson ${ }^{4}$ (D)
}

\begin{abstract}
Observational studies allow researchers to understand the natural history of rheumatic conditions, risk factors for disease development, and factors affecting important disease-related outcomes, and to estimate treatment effect from real-world data. However, this design carries a risk of confounding bias. A propensity score (PS) is a balancing score that aims to minimize the difference between study groups and consequently potential confounding effects. The score can be applied in 1 of 4 methods in observational research: matching, stratification, adjustment, and inverse probability weighting. Systemic lupus erythematosus (SLE) is a rare disease characterized by a relatively small sample size and/or low event rates. In this article, we review the PS methods. We demonstrate application of the PS methods to achieve study group balance in a rare disease using an example of risk of infection in SLE patients with hypogammaglobulinemia.
\end{abstract}

Key Indexing Terms: balancing score, observational, propensity score, selection bias

Clinical research in rheumatology can be complicated by the heterogeneity of many of the systemic autoimmune rheumatic diseases. Observational studies, such as case-control and cohort studies, provide a wider scope of patient representation, lower cost, and longer follow-up time than traditional randomized trials. In addition, observational studies allow researchers to examine potential risk factors for clinically meaningful outcomes. However, these types of studies are criticized for the risk of confounding bias. Confounding of an exposure effect

${ }^{1}$ I. Almaghlouth, MBBS, MSc, Division of Rheumatology, Department of Medicine, University of Toronto, Ontario, Canada, and Rheumatology Unit, Department of Medicine, and College of Medicine Research Center, King Saud University, Saudi Arabia; ${ }^{2}$ E. Pullenayegum, PhD, Division of Biostatistics, Dalla Lana School of Public Health, University of Toronto, and Program in Child Health Evaluative Sciences, SickKids Research Institute, Toronto, Ontario, Canada; ${ }^{3}$ D.D. Gladman, MD, FRCPC, M.B. Urowitz, MD, FRCPC, Division of Rheumatology, Department of Medicine, University of Toronto, and Centre for Prognosis in Rheumatic Diseases, University Health Network, and The Krembil Research Institute, University Health Network, Toronto, Ontario, Canada; ${ }^{4}$ S.R. Johnson, MD, $\mathrm{PhD}$, Division of Rheumatology, Department of Medicine, and Institute of Health Policy, Management and Evaluation, University of Toronto, Toronto, Ontario, Canada.

Dr. S.R. Johnson is supported by a Canadian Institutes of Health Research New Investigator Award.

Dr. S.R. Johnson is a site investigator for scleroderma clinical trials supported by Boehringer Ingelheim, Bayer, Corbus, and GlaxoSmithKline.

Address correspondence to Dr. S.R. Johnson, Toronto Western Hospital, 399 Bathurst Street, Toronto, ON M5T 2S8, Canada.

Email:Sindhu.Johnson@uhn.ca.

Full Release Article. For details see Reprints and Permissions at jrheum.org. Accepted for publication June 12, 2020. requires 2 features: association with the exposure of interest independently from the outcome, and independent association with the outcome but not on the causal pathway of the exposure to the outcome $e^{1,2,3}$. The presence of such a confounder is a threat to the estimated effect of the exposure. Small differences between groups in many variables can accumulate into substantial overall differences ${ }^{1}$. It may be that these differences have a greater effect on the outcome than the intervention itself $f^{4}$. This bias may result in a distortion of the measured treatment effect as a consequence of the way in which the study groups were constructed ${ }^{4}$.

In rheumatic disease research, investigators are also challenged by the rarity of the conditions. A small number of subjects is available for study. Further, the numbers of events may be small. The small sample size can affect the ability to use conventional methodologic and statistical approaches to make inferences about treatment effects or risk estimates ${ }^{1,5,6,7,8,9}$.

In this methodology article, we review propensity score (PS) methods as a potential solution to the risk of bias resulting from confounding, in particular when there are differences between the exposed and nonexposed groups. Specifically, we demonstrate the applicability of PS methods in rheumatic disease studies with small sample size or low event rates, which are commonly encountered in the field of rheumatic diseases research. We highlight 4 PS methods. We discuss the use of standardized differences as a method to evaluate group differences before and after the application of PS methods. We provide an example of how PS methods can be applied using observational data of a rare disease while comparing some of these commonly used methods. The aim of this article is to serve as a guide to clinical researchers who wish to apply PS methods, particularly in the field of rheumatology. 


\section{Propensity score methods}

A propensity score (PS) is a balancing score that can be used to account for the systematic differences between the exposure and control groups in an observational study ${ }^{10}$. The score is constructed by estimating the probability of exposure for each study cohort subject. This is achieved by conditioning the probability of exposure on available observed variables. The PS can be estimated by regressing the treatment assignment on observed baseline characteristics using a logistic regression model (Formula 1$)^{1}$. At an individual level, it is a measure of the likelihood that a person would have been treated considering their baseline characteristics ${ }^{1}$.

$\mathrm{e}(\mathrm{X})=\mathrm{P}(\mathrm{Z}=1 \mid \mathrm{X})$

Formula 1. Propensity score.

$\mathrm{e}(\mathrm{X})=$ propensity score

$\mathrm{Z}=$ exposure, where $1=$ exposed, $0=$ unexposed

$\mathrm{X}=$ a set of baseline characteristics, where $\mathrm{X}=(\mathrm{X} 1$....Xp)

$\mathrm{P}(\mathrm{Z}=1 \mid \mathrm{X})=$ probability of exposure given observed baseline characteristics

Note: Each patient has a probability of exposure where

$0<\mathrm{e}(\mathrm{X})<1$.

The PS can then be used in 1 of 4 methods: matching, stratification, adjustment, and inverse probability weighting (IPW).

Matching. In this method, patients are matched based on their PS using a proximity method with predefined caliper width. This caliper width is based on the SD of the logit of PS ${ }^{1,11}$. Following that, adequacy of matching is assessed using either statistical testing or standardized difference between baseline covariates in the exposure and control groups ${ }^{12}$. The standardized difference is the absolute difference in the sample means divided by an estimate of the pooled SD of the variable. The standardized difference represents the difference in means between the 2 groups in units of SD. A similar formula is used for determining the standardized differences for dichotomous variables ${ }^{12,13}$ (Formula 2 and Formula 3).

$$
d=\frac{\left(\bar{x}_{\text {treatment }}-\bar{x}_{\text {control }}\right)}{\sqrt{\frac{s_{\text {treatment }}^{2}+s_{\text {control }}^{2}}{2}}}
$$

Formula 2. Standardized difference for comparing means. $\mathrm{d}=$ standardized difference

$\bar{x}_{\text {group }}=$ mean of baseline characteristic in the specified group $s_{\text {group }}^{2}=$ variance of baseline characteristic in the specified group

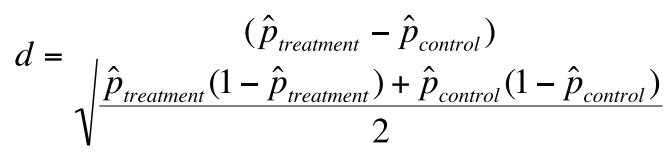

Formula 3. Standardized differences for comparing prevalence.

$\mathrm{d}=$ standardized difference

$\hat{p}_{\text {group }}=$ prevalence of baseline characteristic in the specified group
There is some uncertainty as to what constitutes an optimal standardized difference. Some authors use a standardized difference of 0.1 as the upper limit of acceptable imbalance in baseline covariates, while others divide standardized difference into several cutoffs in which a difference $<0.2$ indicates low imbalance between matching groups, whereas 0.5 is moderate and 0.8 is considered a large imbalance ${ }^{12}$. The utility of the PS was demonstrated by Johnson, et al, where the investigators used matching on PS to improve group balance between patients with systemic sclerosis and pulmonary hypertension who were treated with warfarin compared to those who were not treated with warfarin ${ }^{7}$. The investigators demonstrated that group balance comparable to a randomized trial of similar size was achieved.

The matching method is best used when having a large pool of subjects in a control group. A significant loss of sample size may occur due to the lack of a match. In addition, this method will only account for variables that were included in the construction of the PS. Residual confounding may exist due to the effect of unmeasured confounders ${ }^{12,14}$.

Stratification. Using this method, patients are stratified based on their PS. The exposure group and control groups are compared within each stratum. Wittenborg, et al applied this method to reduce confounding bias in a retrospective cohort study evaluating the use of nonsteroidal antiinflammatory drugs compared to an oral enzyme preparation, thought to have an effect on various rheumatic complaints ${ }^{15}$. Stratification based on PS may be limited by a reduction in sample size within each stratum, which may in turn reduce the power of the study to detect a treatment effect ${ }^{16}$. However, pooling across strata results in power reduction becoming less of an issue.

Adjustment. Using this method, the estimated PS is included in a regression model along with an indicator of exposure assignment. By doing this, within the context of a limited sample size, more confounding variables can be included to create the PS. The application of this technique was demonstrated in a study by Bergstra, et al, in which the authors used PS adjustment when they compared the change in disease activity score in 6 months or 12 months from the initiation of the second treatment regimen of various disease-modifying antirheumatic drugs (DMARD) in patients with rheumatoid arthritis (RA) who initially failed methotrexate (MTX). Patients were divided into categories based on the DMARD that they received after the failure of MTX and the PS was used to adjust for confounding effect in the regression model $^{17}$.

Inverse probability weighting. This unique method uses the PS to create a pseudopopulation in which the exposure is unconfounded. This is achieved by weighting the exposure group based on the inverse of their estimated PS, while weighting the control group based on the inverse of 1 - estimated PS. As a result, all subjects can be used in the study while reducing bias related to the systemic differences between exposure and control subjects (by giving appropriate weight based on estimated PS) ${ }^{10}$. One of the caveats of this method is that it may lead to imprecise estimates if subjects have an extreme estimated PS (i.e., approximate to 0 or 1$)^{18}$. However, there are several proposed mechanisms to 
account for this occurrence, such as using stabilized weight ${ }^{19}$. Finally, the adequacy of balancing groups using this technique can be assessed by comparing the weighted average of the subjects' baseline covariates in both groups ${ }^{10,12,14,16}$.

This method has been used by Kihara, et al to compare the effectiveness of tocilizumab (TCZ) to anti-tumor necrosis factor (anti-TNF) when used as the first biological therapy in patients with RA using data from the British Biological Register. The authors in this study used PS IPW to improve group imbalance between the TCZ and the anti-TNF cohorts ${ }^{20}$.

Small sample sizes. Investigators often question how small the sample size can be to apply PS methods. Pirracchio, et al reported a simulation study evaluating the effect of sample size on the performance of PS matching and IPW methods. They found that reducing the sample size from 1000 subjects to 40 subjects did not significantly affect the type 1 error rate. The IPW method performed better than the PS matching method down to 60 subjects. When the sample size was 40 subjects, the PS matching estimators were either similarly or even less biased than the IPW method estimators ${ }^{21}$.

\section{PS in a rare disease: a demonstration}

Investigators interested in the use of the PS methods often face the challenge of choosing which method to use. This may be particularly challenging in uncommon diseases such as systemic lupus erythematosus (SLE), where the number of subjects available for study may be limited due to rarity of the condition. SLE is a chronic autoimmune disease with a 3-fold higher mortality than general population. Infection is a leading cause of death in this population. Defects in Ig synthesis or function could result in a significant risk of serious infections. We aimed to assess whether acquired low levels of any type of Ig increases the risk of clinically relevant infection in adult patients with SLE ${ }^{22}$.

SLE patients in our long-term, single center, observational cohort were followed at 2- to 6-month intervals according to a standard protocol that included demographics, clinical, laboratory, and therapeutic information ${ }^{22}$. Our study consisted of 437
SLE subjects with low Ig and 656 SLE subjects who never experienced low Ig and served as control subjects. The exposure (low $\mathrm{Ig}$ ) was defined as the presence of 2 low Ig level measurements of the same type with the index date being the first measurement of low Ig. The primary outcome was clinically relevant infection defined as infection within 2 years of the index date requiring use of oral or parenteral antibiotics. The analysis was time to event using a Cox regression model. There were 97 events: 47 in the exposure group and 50 in the control group. Patients with hypogammaglobulinemia had longer mean disease duration (11.2 \pm 9.1 vs $7.6 \pm 8.0 \mathrm{yrs})$, more frequently had a history of lupus nephritis ( $44.9 \%$ vs $17.8 \%)$, higher frequency of proteinuria $(25.6 \%$ vs $11.3 \%)$, and more accumulated SLE damage (mean Systemic Lupus International Collaborating Clinics/ American College of Rheumatology Damage Index score 1.2 \pm 1.6 vs $0.5 \pm 1.0$; Table 1$)^{22}$. Inability to account for these differences between groups would have led to biased estimation of the risk of infection.

We applied 3 PS methods to derive less biased estimates of the risk of infection in SLE patients with low Ig. We applied matching and IPW PS methods separately to investigate our ability to achieve improvement of group balance when comparing the risk of infection between SLE patients with and without acquired low Ig. We favored these 2 methods in particular because of previous studies that demonstrated minimal bias when used to estimate marginal effect ${ }^{23,24}$. We also used PS adjustment due to its usability and the ability to retain the whole cohort. We did not use stratification on the PS because of some criticism about its performance in reducing bias when dealing with few outcome events ${ }^{25}$. Variables used to construct the PS were age, sex, disease duration, disease activity measured by the SLE Disease Activity Index 2000 score, nephrotic range proteinuria, antiphospholipid antibodies, prednisone use and dose, immunosuppressant use, and biologics use ${ }^{26}$. The choice of these covariates was based on the literature regarding associated or predisposing factors to low Ig states. The adequacy of balance was assessed using standardized differences ${ }^{12,19}$.

Table 1. Adequacy of balancing between low Ig and normal Ig groups after using PS in matching and inverse probability weighting.

$$
\begin{gathered}
\text { Normal Ig, } \mathrm{n}=656 \quad \mathrm{n}=437 \quad \begin{array}{c}
\text { STD Diff Before } \\
\text { PS Methods }
\end{array}
\end{gathered}
$$

$37.69 \pm 16.01$
$388(89)$
$7.6 \pm 8.0$
$5.9 \pm 5.9$
$0.5 \pm 1.0$
$74(11.3 \%)$
$168(26.2 \%)$
$349(53.2 \%)$
$15.3 \pm 14.6$
$152(23.2 \%)$

$1(0.2 \%)$

$42.37 \pm 14.10$
$570(87)$
$11.2 \pm 9.1$
$6.2 \pm 6.3$
$1.2 \pm 1.6$
$112(25.6 \%)$
$62(15.2 \%)$
$332(76.0 \%)$
$16.8 \pm 16.8$
$201(46.0 \%)$

$5(1.1 \%)$

\begin{tabular}{ccc}
0.19 & 0.14 & 0.176 \\
0.06 & 0.04 & 0.004 \\
0.43 & 0.21 & 0.334 \\
0.05 & 0.04 & 0.024 \\
0.59 & 0.32 & 0.46 \\
0.39 & 0.18 & 0.29 \\
0.25 & 0.26 & 0.17 \\
0.48 & 0.14 & 0.31 \\
0.32 & 0.1 & 0.20 \\
0.5 & 0.17 & 0.36 \\
0.13 & 0.01 & 0.09 \\
\hline
\end{tabular}

Values are mean \pm SD or $\mathrm{n}(\%)$. APA: antiphospholipid antibody; IPW: inverse probability weighting; PS: propensity score; SDI: Systemic Lupus International Collaborating Clinics/American College of Rheumatology Damage Index; SLEDAI-2K: Systemic Lupus Erythematosus Disease Activity Index 2000; STD diff: standardized differences. 
Both PS matching and the IPW improved group balance (Table 1). Matching by PS demonstrated superior improvement in the standardized difference 8 of 11 (73\%) of the variables. However, matching by PS resulted in smaller sample size (from 1093 subjects in the unmatched cohort to 922 subjects in the matched cohort) due to the loss of unmatched subjects. In comparison, the IPW was able to improve balance across all the variables. In addition, it allowed retention of the whole cohort $(\mathrm{n}=1093)$. Adjustment by PS was also applied and allowed for retention of the complete cohort. However, this method did not allow for evaluation of reduction in group imbalances.

Comparison of estimates of the risk of infection in SLE patients with and without low Ig using the 3 PS methods are presented in Table 2.

All 3 PS methods demonstrated that a low IgA level significantly increased the risk of infection in patients with SLE. Adjustment by PS had the greatest uncertainty around the estimate of risk (HR 3.19, 95\% CI 1.17-8.71). PS matching and IPW gave estimates of comparable magnitude and uncertainty, with IPW giving the most conservative estimate (HR 1.75, 95\% CI 1.01-3.02; Table 2).

This example illustrates the application of the PS methods. It was previously believed that the PS methods could only be used in large administrative databases. These methods are increasingly being successfully applied in observational data of rare diseases ${ }^{7,27,28}$. Further, our study provides a comparison between several PS methods performances in reducing groups imbalance when applied to a survival model-based study with relatively small event rate. The robustness of the PS matching and inverse probability of treatment weighting methods in reducing potential bias due to measured confounders in our study was largely consistent with the simulation study by Pirracchio, et al, in which the authors demonstrated good performance of both techniques when the sample size was as low as 40 subjects $^{21}$.

\section{Conclusion}

In this paper, we have described the use of PS methods to reduce the risk of bias in estimates of treatment effect or risk using observational data. We have highlighted their relative advantages and disadvantages. We have demonstrated the successful use of these methods in observational data of a rare disease, evaluating the risk of infection in SLE patients with low Ig. Rheumatic disease researchers may consider working with biostatisticians to apply the PS methods to observational studies of rare rheumatic diseases.

Table 2. Comparison of estimates of risk of infection in SLE patients with and without low IgA using 3 propensity score methods.

\begin{tabular}{lcc}
\hline & \multicolumn{2}{c}{ Low IgA } \\
\hline Propensity Score Method & HR & $95 \%$ CI \\
Matching & 2.24 & $1.61-3.12$ \\
Inverse probability weighting & 1.75 & $1.01-3.02$ \\
Adjustment & 3.19 & $1.17-8.71$ \\
\hline
\end{tabular}

SLE: systemic lupus erythematosus.

\section{REFERENCES}

1. Johnson SR, Tomlinson GA, Hawker GA, Granton JT, Feldman BM. Propensity score methods for bias reduction in observational studies of treatment effect. Rheum Dis Clin N Am 2018;44:203-13.

2. Johnston R, Jones K, Manley D. Confounding and collin earity in regression analysis: a cautionary tale and an alternative procedure, illustrated by studies of British voting behaviour. Qual Quant 2018;52:1957-76.

3. VanderWeele TJ, Shpitser I. On the definition of a confounder. Ann Stat 2013;41:196-220.

4. Savitz DA. Interpreting epidemiologic evidence. Strategies for study design and analysis. Oxford: Oxford University Press; 2003.

5. Johnson SR. Bayesian inference: Statistical gimmick or added value? J Rheumatol 2011;38:794-6.

6. Johnson SR, Feldman BM, Pope JE, Tomlinson GA. Shifting our thinking about uncommon disease trials: the case of methotrexate in scleroderma. J Rheumatol 2009;36:323-9.

7. Johnson SR, Granton JT, Tomlinson GA, Grosbein HA, Le T, Lee $\mathrm{P}$, et al. Warfarin in systemic sclerosis-associated and idiopathic pulmonary arterial hypertension. A bayesian approach to evaluating treatment for uncommon disease. J Rheumatol 2012;39:276-85.

8. Johnson SR. Advanced epidemiologic methods for the study of rheumatic and musculoskeletal diseases. Rheum Dis Clin N Am 2018;44:xv-xvi.

9. Johnson SR, Tomlinson GA, Granton JT, Hawker GA, Feldman BM. Applied Bayesian methods in the rheumatic diseases. Rheum Dis Clin N Am 2018;44:361-70.

10. Rosenbaum PR, Rubin DB. The central role of the propensity score in observational studies for causal effects. Biometrika 1983;70:41-55.

11. Austin PC, Grootendorst P, Anderson GM. A comparison of the ability of different propensity score models to balance measured variables between treated and untreated subjects: a Monte Carlo study. Stat Med 2007;26:734-53.

12. Austin PC. Balance diagnostics for comparing the distribution of baseline covariates between treatment groups in propensity-score matched samples. Stat Med 2009;28:3083-107.

13. Austin PC. Assessing balance in measured baseline covariates when using many-to-one matching on the propensity-score. Pharmacoepidemiol Drug Saf 2008; 17:1218-25.

14. Austin PC. A tutorial and case study in propensity score analysis: An application to estimating the effect of in-hospital smoking cessation counseling on mortality. Multivar Behav Res 2011;46:119-51.

15. Wittenborg A, Bock PR, Hanisch J, Saller R, Schneider B. Comparative epidemiological study in patients with rheumatic diseases illustrated in a example of a treatment with non-steroidal anti-inflammatory drugs versus an oral enzyme combination preparation. [Article in German] Arzneimittelforschung 2000;50:728-38.

16. Austin PC. The relative ability of different propensity score methods to balance measured covariates between treated and untreated subjects in observational studies. Med Decis Making 2009; 29:661-77.

17. Bergstra SA, Winchow LL, Murphy E, Chopra A, Salomon-Escoto $\mathrm{K}$, Fonseca JE, et al. How to treat patients with rheumatoid arthritis when methotrexate has failed? The use of a multiple propensity score to adjust for confounding by indication in observational studies. Ann Rheum Dis 2019;78:25-30.

18. Williamson E, Morley R, Lucas A, Carpenter J. Propensity scores: from naive enthusiasm to intuitive understanding. Stat Methods Med Res 2012;21:273-93.

19. Austin PC, Stuart EA. Moving towards best practice when using inverse probability of treatment weighting (IPTW) using the propensity score to estimate causal treatment effects in observational studies. Stat Med 2015;34:3661-79. 
20. Kihara M, Davies R, Kearsley-Fleet L, Watson KD, Lunt M, Symmons DP, et al; British Society for Rheumatology Biologics Register. Use and effectiveness of tocilizumab among patients with rheumatoid arthritis: an observational study from the British Society for Rheumatology Biologics Register for rheumatoid arthritis. Clin Rheumatol 2017;36:241-50.

21. Pirracchio R, Resche-Rigon M, Chevret S. Evaluation of the propensity score methods for estimating marginal odds ratios in case of small sample size. BMC Med Res Methodol 2012;12:70.

22. Almaghlouth IS, Pullenayegum E, Johnson S, Gladman DD, Urowitz M. Exploring the relation between immunoglobulins level and infection risk in adult patients with systemic lupus erythematosus [abstract]. Arthritis Rheumatol 2018;70 Suppl 10.

23. Austin PC. The performance of different propensity score methods for estimating marginal hazard ratios. Stat Med 2013;32:2837-49.

24. Austin PC. The use of propensity score methods with survival or time-to-event outcomes: reporting measures of effect similar to those used in randomized experiments. Stat Med 2014;33:1242-58.
25. Elze MC, Gregson J, Baber U, Williamson E, Sartori S, Mehran $\mathrm{R}$, et al. Comparison of propensity score methods and covariate adjustment: evaluation in 4 cardiovascular studies. J Am Coll Cardiol 2017;69:345-57.

26. Gladman D, Ginzler E, Goldsmith C, Fortin P, Liang M, Urowitz $\mathrm{M}$, et al. The development and initial validation of the Systemic Lupus International Collaborating Clinics/American College of Rheumatology Damage Index for systemic lupus erythematosus. Arthritis Rheum 1996;39:363-9.

27. Urowitz MB, Ohsfeldt RL, Wielage RC, Kelton KA, Asukai Y, Ramachandran S. Organ damage in patients treated with belimumab versus standard of care: a propensity score-matched comparative analysis. Ann Rheum Dis 2019;78:372-9.

28. Urowitz MB, Su J, Gladman DD. Atherosclerotic vascular events in systemic lupus erythematosus: an evolving story. J Rheumatol 2020;47:66-71. 